\title{
Three Dimensional Visualization Of Proteins In Cellular Interactions
}

\author{
Colin R. F. Monks ${ }^{1}$ Patricia J. Crossno ${ }^{2}$ George Davidson ${ }^{3}$ \\ Constantine Pavlakos ${ }^{3}$ Abraham Kupfer ${ }^{1}$ \\ Cláudio Silva ${ }^{4} \quad$ Brian Wylie $^{2}$ \\ ${ }^{1}$ Division of Basic Science, Department of Pediatrics, National Jewish Center for Immunology \\ and Respiratory Medicine \\ ${ }^{2}$ Computer Science Department, University of New Mexico \\ ${ }^{3}$ Massively Parallel Computer Research Laboratory, Sandia National Laboratories \\ ${ }^{4}$ Computer Science Department, State University of New York at Stony Brook
}

\begin{abstract}
In researching the communication mechanisms between cells of the immune system, visualization of proteins in three dimensions can be used to determine which proteins are capable of interacting with one another at a given time by showing their spatial colocality. Volume data sets are created using digital confocal immunofluorescence microscopy. A variety of visualization approaches are then used to examine the interactions. These include volume rendering, isosurface extraction, and virtual reality. Based on our experiences, we have concluded that no single one of these approaches provides a complete solution for visualizing biological data. However, in combination, their respective strengths complement one another to provide an understanding of the data.
\end{abstract}

\section{INTRODUCTION}

One of the most common tools in modern biology is the light microscope, which traditionally has been used to do 2dimensional imaging of various living systems. One of the most useful permutations of this technology is immunofluorescence microscopy, which allows researchers to visualize the location of individual proteins within the cell. With the recent development of confocal technology, immunofluorescence microscopy now has the capability of imaging proteins in three dimensions. Previously, visualization techniques within biology did not permit one to examine the relative locations of various proteins in three dimensions. This 3-dimensional information is important for finding which proteins interact by establishing that they are present at the same time in the same location. Using the methods presented here, we can now establish the colocality of proteins during cell/cell interactions.

\section{CELL / CELL INTERACTION}

Communication between cells is of great importance throughout biology. This communication often takes place through direct cell/cell interactions, where cells physically come in contact with one another. Cell/cell interactions are of primary importance in organism development, immune system responses, etc.

\section{IMMUNOFLUORESCENCE MICROSCOPY}

The subcellular distribution of individual proteins can be observed at the light microscope level (i.e. 100 nm) using a technique called immunofluorescence microscopy. Antibodies are generated to the specific protein one wishes to observe. Cell conjugates are formed by mixing the cells together. The conjugates are plated on coverslips, fixed to preserve structure, detergent extracted to permeabilize membranes, and incubated with the appropriate antibody [4]. Fluorescently tagged secondary antibodies are then used to visualize the location of the protein one wishes to observe.

\section{VOLUME CREATION}

Using 3-dimensional digital confocal immunofluorescence microscopy, we investigated the localization of a number of proteins during cell/cell interactions. Series of 3-dimensional immunofluorescent images were collected using a Zeiss Axiophot microscope, a SpectraSource CCD camera, and a high resolution stepper motor. The image sets were subsequently deconvolved by constrained iterative deconvolution on an Intel Paragon supercomputer.

\section{DECONVOLVING THE IMAGES}

The microscope and camera collect light from fluorescent molecules that lie both in, and out of the focal plane of the objective lens. This mixing of light from regions above and below the focal plane is one of the main reasons 3-dimensional immunofluorescent microscopy is difficult. However, the physics describing this phenomenon is understood and computational algorithms are available to correctly redistribute measured light to its proper origin (note that this approach is not image processing to artificially improve the image, but is based on the underlying physics).

The objective lens in the microscope spreads a point source of light into a blur. This blurring is characterized by the Point Spread Function, PSF, of the lens. The observed images are the result of convolving that PSF with the actual 3-dimensional light intensities in the sample.

An estimate of the actual 3-dimensional intensities can be found by deconvolving the observed images using the measured (or less ideally, the theoretical) PSF of the lens. Unfortunately, direct deconvolution is extremely sensitive to measurement noise and is not a good algorithm for practical applications. Instead of direct deconvolution, we follow David Agard who gives an iterative algorithm [1], which gradually deconvolves the image while enforcing the physi- 


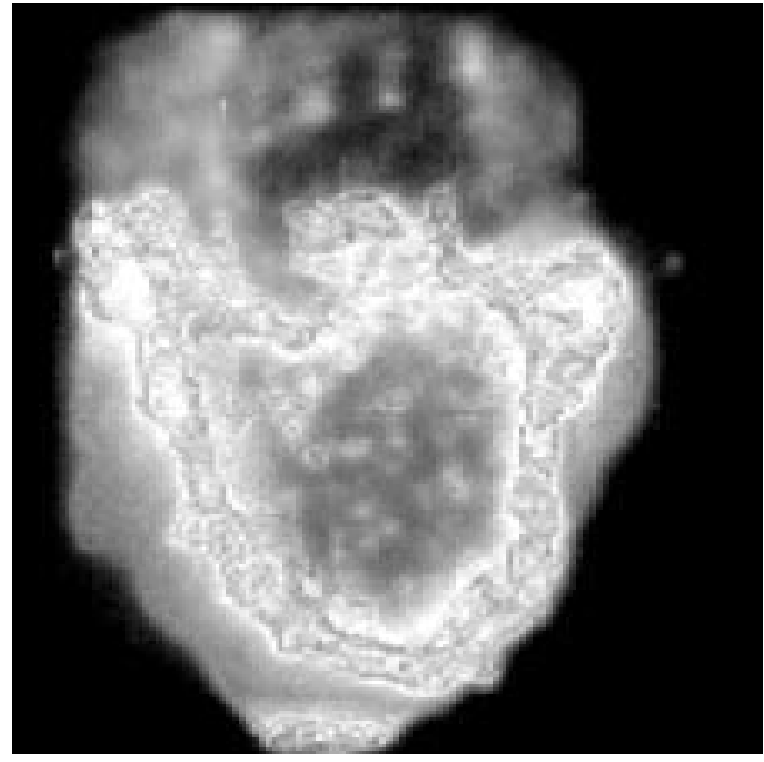

Figure 1: Volume rendering of single cell.

cal constraint that every spacial point must have non-negative light intensity. The deconvolution is very computationally intense, even when using FFTs, but an even more pressing problem is to provide enough memory for the data structures required to deconvolve large (e.g., $1024 \times 1024 \times 64)$ data sets. This requirement was the initial reason for our collaboration between Sandia and the National Jewish Center. The visualization techniques reported here improve our understanding of the 3-dimensional structures revealed by images sharpened by deconvolution.

\section{VISUALIZATION APPROACHES}

We visualized cell/cell interactions using both volume rendering and isosurface extraction. The isosurfaces were then rendered and viewed using either AVS or an interactive exploration tool developed at Sandia called EIGEN/VR, which allows data analysis from within a virtual reality environment.

\section{Volume Rendering}

As a technique, volume rendering [3] accumulates information from all voxels in the data set to produce an image, enabling a comprehensive examination of the global structure in a data set. The technique works by modeling the volumetric data set (in our case the cell data) as a cloud-like material that scatters, emits and absorbs light [6]. Roughly speaking, for each ray, the rendering equation $\int e^{-\int_{0}^{x} \sigma(t) d t} \cdot I(x) d x$ is integrated, where $I(x)$ represents the intensity of light emanating from a given portion of the volume and $\sigma(t)$ is the differential absorption of light (used to calculate attenuation along the viewing direction). In our

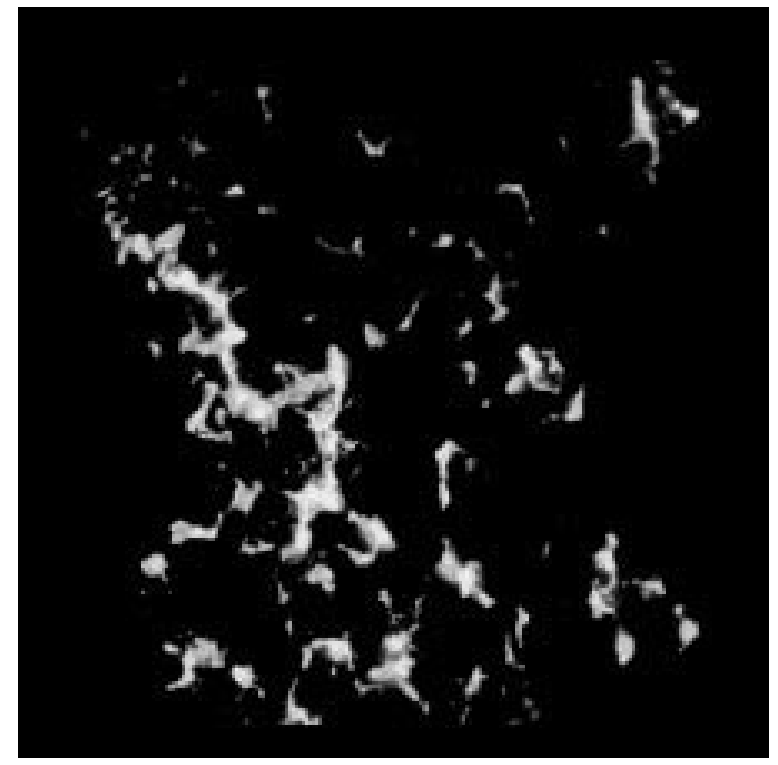

Figure 2: Thymic gland tissue showing the thymic epithelial cells.

renderer, we use the so called transfer functions to specify what portions of the volume are relevant to be visualized. Transfer functions are just like color maps, they specify color (RGB) and opacity for each voxel in the volume.

In looking for interesting properties in the data, it is imperative to be able to try different combinations of transfer functions and viewing parameters. In cases where the data is very complex, one effective way to see patterns in the data is by using animations. Our eyes are very well trained to extract 3-dimensional information from animations (such as rotations). Unfortunately, volume rendering is typically very slow, even for small data sets, and especially when the volume is relatively transparent (our case).

We started out trying to visualize our data using VolVis [2], a state-of-the-art volume visualization system developed at Stony Brook, but it took from several minutes to several hours to generate animations of our smaller data sets. Performing visualization in such a manner is of limited use as it is counter-productive to have to wait hours for animations that might not contain useful information.

By using the new Parallel Volume Rendering system (PVR), being developed under a collaboration between Sandia National Laboratories and the State University of New York at Stony Brook, on Sandia's 1800-node Intel Paragon, we are able to generate even the largest animations in a few seconds to a few minutes as the system is easily scalable to the desirable performance (e.g. the larger the data set, the more nodes we use; the same goes for large animations). PVR is meant to provide interactive visualization of large volumetric data sets in parallel and distributed environments. PVR was used to visualize a single cell, Figure 1, as well as a sampling of Thymus gland tissue showing the thymic epithelial cells, Figure 2. The tissue volume was relatively large at 1024 x 1024 x 64 voxels - an animation was produced in just a few minutes using PVR on the Paragon. 


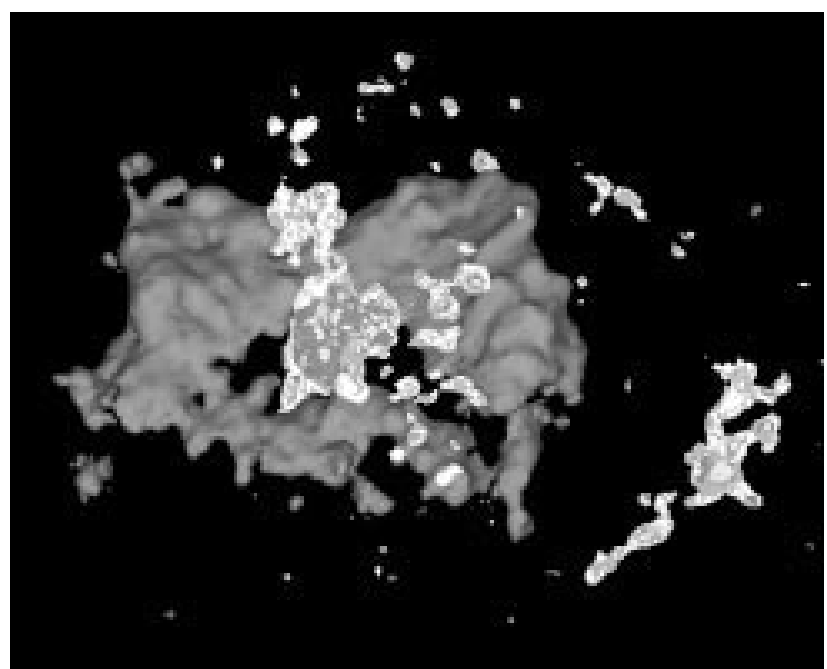

Figure 3: Isosurfaces of two proteins, one in red, one in green, with darker focus regions.

\section{Isosurface Extraction}

An isosurface is a single-valued contour in 3-space. It is the surface that partitions a volume into those locations whose sample values lie on or above a selected surface value (threshold) and those locations whose values lie below it. Consequently, isosurface extraction reduces the volume data set to just those values selected by the researcher.

In our examples, each volume data set was thresholded using a parallel implementation of the marching cubes algorithm [5] combined with triangle decimation [7] running on the Intel Paragon. The data sets are registered so that surfaces from multiple volumes can be combined and the spacial relationships between the proteins can be seen.

In Figure 3, we threshold each volume a second time to produce a smaller, more concentrated region, which we call the focus. The smaller, darker, more opaque surfaces are the focus regions, and the larger, lighter, transparent surfaces are the less concentrated regions of each protein. Color is used to correlate the types of proteins.

However, in Figure 4, it was difficult to distinguish the intersection of the two focus regions through the multiple layers of transparency, so we replaced them with a single surface, shown in yellow, representing their intersection. The intersection surface was created by generating a volume that combined the two data sets using the two focus thresholds. Voxels where either of the two source volumes were below their respective thresholds were zeroed out in the combination volume. Otherwise, the voxels were set to the sum of the voxel values. The resulting volume was then input to marching cubes with a threshold set to the sum of the two focus thresholds.

\section{Virtual Reality Presentation}

EIGEN/VR uses the technology of immersive environments (or "Virtual Reality") to provide a sophisticated humancomputer interface for the exploration of complex 3-dimensional data. Once certain isosurfaces were extracted from

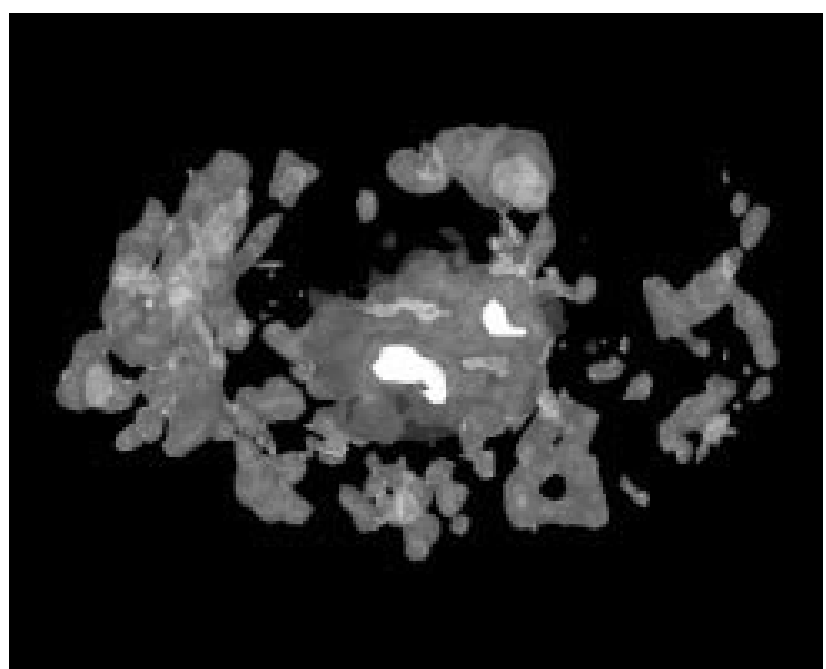

Figure 4: Isosurfaces of two proteins, one in red, one in green, with the intersection of their focus regions in yellow.

the volumetric data, they were imported into EIGEN/VR for further study. The ability to immerse oneself within a true 3dimensional presentation of the data is particularly useful for understanding the 3-dimensional structural intricacies in the data. This capability is impossible to convey using 2dimensional media - however, an example image from EIGEN/VR is shown in Figure 5. A current shortcoming of this technology is the inability to experience volume renderings in a similar manner due to the extensive nature of the computations needed to generate each view.

\section{RESULTS}

Three-dimensional imaging reveals that different proteins can reside in distinct locations within cell conjugates. For example, one protein shown in green in Figure 3, concentrates in a small area. However, a different protein shown in red is found in a surrounding region. In Figure 4, the green represents the same protein as in Figure 3, but the red is a different protein that localizes to a region which includes, but is larger than, the first protein. Note that the yellow region shows where both proteins colocalize. This is important because only proteins which are spatially together can interact. Knowledge of this kind provides an important tool for studying molecular interactions within cells.

The rendering of thymic epithelial cells shows that they are distributed within a complicated, yet resolvable, 3dimensional network within the thymus. This information can be used in the future in multiple protein labeling experiments to explore cellular interactions within this tissue.

\section{CONCLUSIONS}

This work has resulted in significant technology for enabling the understanding of microscopic biological systems in three dimensions. In this case, this technology has been applied to further a better understanding of the physical attributes associated with cell communications. Our collaboration, and the resulting virtual laboratory that has been 


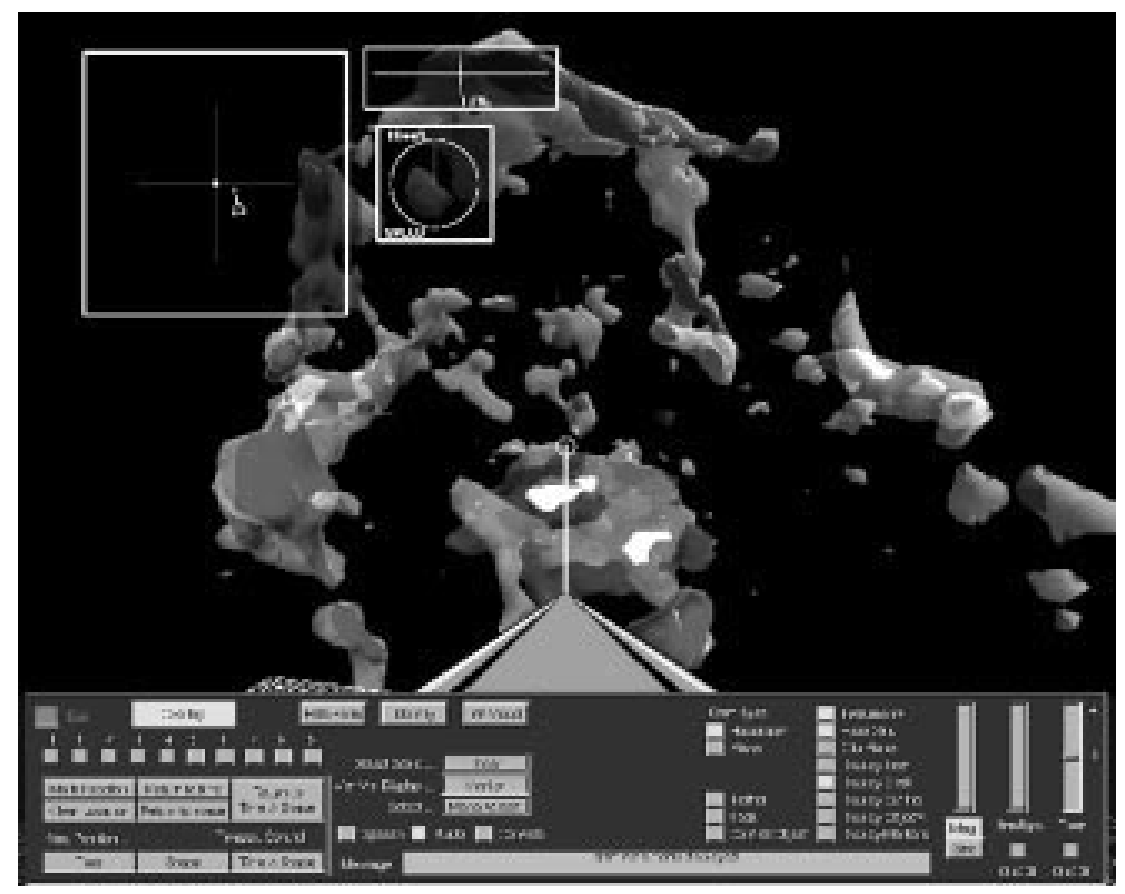

Figure 5: EIGEN/VR interface exploring Figure 4 data.

formed, provides a clear example where the whole exceeds the sum of the parts.

For best results, we found that a combination of various visualization approaches was needed. Volume rendering provides a view of the whole cell's structure along with the relative intensities within the cell - this global information offers insights towards more detailed feature-based investigations. Isosurfaces permit features such as the spatial relationships between proteins to be seen more cleanly. Interactive viewing using virtual-reality technologies further enhances the 3-dimensional comprehension of the data. All of these approaches complement each other nicely to allow a thorough visual understanding.

\section{ACKNOWLEDGEMENTS}

This research was funded by the Applied Mathematical Sciences program, U.S. DOE, Office of Energy Research. The work was performed at Sandia National Laboratories, operated for DOE under contract No. DE-AC04-76DP00789. Cláudio Silva was supported during his Ph.D. by CNPqBrasil, NSF and Sandia National Labs. We thank Arie Kaufman and Maurice Fan Lok for their work on the PVR system. The studies at NJCIRM were supported in part by grant AI-23764 (to A.K.) from the National Institutes of Health.

\section{ELECTRONIC INFORMATION}

For animations of the data sets shown in the figures and more information on Sandia's data visualization projects see <http://www.cs.sandia.gov/VIS > on the web. The National Jewish Center's web location concerning this research is <http://www.njc.org/profinfohtml/SC95.html>.

Our email addresses are as follows: Colin Monks (monksc@njc.org), Patricia Crossno (pjcross@cs.sandia.gov), George Davidson (gsdavid@cs.sandia.gov), Constantine Pavlakos (cjpavla@cs.sandia.gov), Avi Kupfer (kupfera@njc.org), Cláudio Silva (csilva@cs.sunysb.edu), and Brian Wylie (bnwylie@cs.sandia.gov).

\section{REFERENCES}

[1] Agard, D. A., "Optical Sectioning Microscopy: Cellular Architecture in Three Dimensions," Annual Review of Biophysics and Bioengineering, pp. 191-219, 1984.

[2] Avila, R., T. He, L. Hong, A. Kaufman, H. Pfister, C. Silva, L. Sovierajski, and S. Wang, "VolVis: A Diversified Volume Visualization System," IEEE Visualization '94, pp. 85-92, October 1994.

[3] Kaufman, A., "Volume Visualization," IEEE Computer Society Press, 1990.

[4] Kupfer, H., C. R. Monks, and A. Kupfer, "Small splenic $\mathrm{B}$ cells that bind to antigen-specific $\mathrm{T}$ helper (Th) cells and face the site of cytokine production in the Th cells selectively proliferate: immunofluorescence microscopic studies of Th- B antigen-presenting cell interactions," J. Exp. Med., 179, pp. 1507-15, 1994.

[5] Lorensen, W. E., and H. E. Cline, "Marching Cubes: A High Resolution 3D Surface Construction Algorithm," Computer Graphics, Vol. 21, No. 4, (SIGGRAPH '87), pp. 163-169, July 1987.

[6] Max, N.,"Optical Models for Direct Volume Rendering," IEEE Trans. Visualization and Computer Graphics, Vol. 1, No. 2, pp. 99-108, June 1995.

[7] Schroeder, W. J., J. A. Zarge, and W. E. Lorensen, "Decimation of Triangle Meshes," Computer Graphics, Vol. 26, No. 2 (SIGGRAPH ‘92), pp. 65-70, July 1992. 